\title{
Calidad de vida global en personas con demencia
}

\author{
Ramona Lucas-Carrasco, Maribel Peró, Jaume March
}

Objetivo. Evaluar la calidad de vida (CV) global en personas con demencia e investigar las variables asociadas con la percepción subjetiva de la CV.

Pacientes y métodos. Se llevó a cabo un estudio transversal en seis centros de Barcelona y Tarragona. Un total de 99 personas con demencia leve-moderada que vivían en casa con un cuidador conocido completaron una entrevista cara-cara que incluía información sociodemográfica, percepción del estado de salud, un listado de problemas crónicos de salud, sintomatología depresiva, estado funcional, aspectos positivos, sentido de la vida y satisfacción con las relaciones personales. La CV global se evaluó con la pregunta global del World Health Organization Quality of Life-BREF.

Resultados. El 40,6\% de los entrevistados consideró su CV como buena/muy buena. Los análisis univariados de regresión logística ordinal y binaria no mostraron asociación entre CV con datos sociodemográficos, gravedad de la demencia ni estado funcional, pero sí con las otras variables de estudio. Los análisis multivariados de la regresión logística ordinal y binaria mostraron que una mejor percepción de salud y mayor satisfacción con las relaciones personales se asociaban con mejor CV. Asimismo, en el análisis multivariado de regresión logística ordinal, mejor CV se asoció con un menor número de síntomas depresivos, y en el análisis de regresión logística binaria, con disfrutar de la vida y menor gravedad de la demencia.

Conclusiones. Personas con demencia leve-moderada pueden informar sobre su CV y estados subjetivos. Además de los aspectos relacionados con la salud, aspectos positivos y las relaciones personales deberían evaluarse sistemáticamente en esta población.

Palabras clave. Autopercepción. Calidad de vida. Demencia. Estado de salud.

\section{Introducción}

La demencia es un síndrome caracterizado por un deterioro global progresivo de la función intelectual, y una de las principales causas de discapacidad en las personas mayores [1]. En el momento actual, no existe un tratamiento que cure la demencia; por ello, la atención se centra en promover el bienestar y la calidad de vida (CV) [2-4]. La evaluación de la $\mathrm{CV}$ es tan importante como la evaluación del deterioro cognitivo, de las alteraciones conductuales o del estado funcional $[5,6]$. La CV refleja una mayor valoración de cómo se siente la persona y del grado de satisfacción con una determinada intervención o tratamiento [7].

La CV es un constructo multidimensional que incluye varias áreas: física, psicológica y social [8,9]; la evaluación debe ser subjetiva y debe incluir aspectos positivos y negativos [3,9]. Además, la CV en personas con demencia debería incluir facetas específicas $[3,10]$. Durante la última década, ha habido un interés considerable por el estudio de la CV en personas con demencia [11]. Un mayor deterioro cog- nitivo, peor estado funcional $y$, de forma concluyente, la depresión se asocian con peor CV $[2,3,6,10]$.

Los estudios que comparan la CV autoevaluada en personas con demencia con la CV evaluada por un cuidador muestran de forma sistemática que los cuidadores evalúan la $\mathrm{CV}$ peor que las propias personas con demencia [3,10,12-14]. Las diferencias en las puntuaciones se observan en áreas específicas, y además están influidas por el grado de relación entre el cuidador y la persona con demencia [12-14]. Por tanto, la evaluación de la CV realizada por personas con demencia y por sus cuidadores puede diferir de forma sustancial.

Existe cada vez mayor evidencia de que las personas con demencia leve-moderada proporcionan información apropiada de sus propios estados subjetivos $[3,10,15,16]$. En la práctica clínica, la evaluación a través de una única pregunta puede ser una forma eficiente y útil de medir la $\mathrm{CV}$ en personas con demencia [17]. El objetivo principal de este estudio fue evaluar la percepción de la CV en personas con demencia leve-moderada, utilizando la pregunta de CV global del cuestionario WHOQOL
CRE Alzheimer (R. Lucas-Carrasco); Salamanca. Departamento de Metodología; Facultad de Psicología; Universitat de Barcelona (R. Lucas-Carrasco, M. Peró); Barcelona. Institut de Recerca Biomèdica de Lleida (J. March); Lleida, España.

Correspondencia: Dra. Ramona Lucas Carrasco. Departamento de Metodología. Facultad de Psicología. Universitat de Barcelona. Pg. Vall d'Hebron, 171. E-08035 Barcelona.

Fax: +34934021359.

E-mail:

lucas.ramona@gmail.com

Financiación:

R.L.C. y J.M. son miembros del Grupo Consolidado de Investigación 2009SGR822 de la Generalitat de Catalunya.

Agradecimientos:

A la Dra. Betty S. Black (Johns Hopkins Medical Institutions, Baltimore, MD, Estados Unidos), por la revisión y comentarios del manuscrito.

Datos parciales del artículo se presentaron en el XIV Congreso Mundial de Psiquiatría (Munich, Alemania, 27 febrero-2 marzo de 2010).

Aceptado tras revisión externa: 17.11.10.

Cómo citar este artículo: Lucas-Carrasco R, Peró M, March J. Calidad de vida global en personas con demencia. Rev Neurol 2011; 52: $139-46$.

(C) 2011 Revista de Neurología 
(World Health Organization Quality of Life)-BREF: 'En general, ¿cómo puntuaría su calidad de vida?,' así como investigar las variables asociadas con ésta. Basándonos en resultados de estudios previos [3,10, 15-17], nuestra hipótesis fue que las personas con demencia leve-moderada serían capaces de responder a la pregunta general sobre su $\mathrm{CV}$ y que -además de la gravedad de la demencia y variables relacionadas con la salud- aspectos positivos y la satisfacción con las relaciones personales estarían asociadas con la CV.

\section{Pacientes y métodos}

\section{Muestra}

Se utilizó una muestra de conveniencia. Los participantes se reclutaron de seis centros en Barcelona y Tarragona (tres unidades de diagnóstico, dos hospitales de día y un centro de día). Los criterios de inclusión fueron personas con diagnóstico de demencia (Manual diagnóstico y estadístico de los trastornos mentales, $4 .^{a}$ edición, DSM-IV) [18], en fase leve-moderada (Mini Mental State Examination, MMSE $\geq 10$ ) [19], que vivieran en casa y tuvieran un cuidador.

Se proporcionó una descripción del protocolo de estudio. En cada centro, una persona colaboradora con los investigadores del estudio invitó a las personas con demencia y sus cuidadores a participar. A pesar de que los cuidadores no aportaron datos para este estudio, fueron informados e invitados a participar por razones éticas, dado que no todos los participantes conocían su diagnóstico; por esta razón, en el protocolo de estudio se utilizó la palabra 'deterioro cognitivo' en lugar de 'demencia' [20]. El estudio fue aprobado por el Comité de Ética de la Universitat Autònoma de Barcelona. Todos los participantes firmaron la hoja de consentimiento informado. Toda la información, autocomunicada, se recogió mediante una entrevista personal llevada a cabo por un psicólogo o neuropsicólogo previamente entrenado.

\section{Medidas}

\section{Demencia}

El diagnóstico de demencia (DSM-IV) [18] se obtuvo mediante la revisión de la historia clínica.

\section{Calidad de vida global}

Se evaluó mediante la pregunta global de CV del WHOQOL-BREF [21] en su versión española [22]: 'En general, ¿cómo puntuaría su calidad de vida?, con cinco opciones de respuesta: muy buena, buena, normal, mala o muy mala. El WHOQOL-BREF es un cuestionario genérico de $\mathrm{CV}$ compuesto por 24 preguntas agrupadas en cuatro áreas (física, psicológica, relaciones sociales y ambiente) y dos preguntas generales sobre $\mathrm{CV}$ y salud.

\section{Indice de Barthel $[23,24]$}

Es una medida que valora el estado funcional o grado de independencia para realizar actividades básicas de la vida diaria. Puntuaciones mayores indican mayor grado independencia. Se utilizó un punto de corte de 55 para dividir a los participantes en personas con dependencia leve/ninguna (índice de Barthel, IB $\geq 55$ ) y con dependencia moderada/grave $(\mathrm{IB}<55)[24]$.

\section{Escala de depresión geriátrica [25]}

Se utilizó la versión española de 15 preguntas [26] de la Geriatric Depression Scale (GDS). La GDS-15 mide sintomatología depresiva y se contesta en una escala dicotómica 'sí/no'; puntuaciones menores indican menor sintomatología depresiva. Se utilizó un punto de corte de 6 para dividir a los participantes en personas sin depresión (GDS-15<6) y con depresión (GDS-15 $\geq 6$ ) [25].

\section{Test minimental de Folstein (MMSE) [19]}

La gravedad de la demencia se evaluó mediante el MMSE. Puntuaciones más bajas indican mayor grado de deterioro cognitivo. Se utilizó un punto de corte de 20 para dividir la muestra en personas con demencia leve (MMSE $>20$ ) y moderada (MMSE $\leq 20)$.

\section{Percepción subjetiva del estado de salud}

Se obtuvo preguntando a los participantes sobre su estado de salud: 'En general, usted diría que su salud es excelente, muy buena, buena, regular o mala, y un listado de problemas crónicos de salud: hipertensión arterial; problemas de corazón; pulmón; estómago o intestino; neurológicos; renales; de tiroides; artrosis, problemas de huesos y articulaciones; diabetes; depresión; cáncer; problemas de audición y de visión. Los participantes contestaban si los tenían o no.

\section{Cuestionario WHOQOL}

Se seleccionaron tres facetas de CV correspondientes al cuestionario WHOQOL [9]; facetas que hasta ahora se habían tenido menos en cuenta en personas con demencia:

- Una faceta relacionada con aspectos positivos: ¿cuánto disfruta de la vida?

- Una faceta del área espiritual/creencias: ¿siente 
Tabla I. Información sociodemográfica y análisis univariado.

\begin{tabular}{|c|c|c|c|c|c|}
\hline & $\begin{array}{c}\mathrm{CV} \mathrm{mb} / \mathrm{b}, n=40 \\
\text { (\% fila) }\end{array}$ & $\begin{array}{c}\mathrm{CV} n / \mathrm{m}, n=59 \\
\text { (\% fila) }\end{array}$ & $\begin{array}{c}\text { Muestra total, } n=99 \\
\text { ( } \% \text { columna) }\end{array}$ & $\begin{array}{l}\text { Regresión logística ordinal } \\
\text { Odds ratio (IC 95\%) }\end{array}$ & $\begin{array}{l}\text { Regresión logística binaria } \\
\text { Odds ratio (IC 95\%) }\end{array}$ \\
\hline Edad media \pm desviación estándar & $79,2 \pm 6,7$ & $78,5 \pm 7,8$ & $78,7 \pm 7,3$ & $1,03(0,98-1,09)$ & $1,01(0,95-1,07)$ \\
\hline \multicolumn{6}{|l|}{ Sexo } \\
\hline Mujer & $22(34,9)$ & $41(65,1)$ & $63(63,6)$ & $C R$ & CR \\
\hline Varón & $18(50,0)$ & $18(50,0)$ & $36(36,4)$ & $1,37(0,6-2,9)$ & $1,86(0,8-4,3)$ \\
\hline \multicolumn{6}{|l|}{ Estado civil } \\
\hline Viudo/a & $20(37,0)$ & $34(63,0)$ & $54(54,5)$ & $C R$ & CR \\
\hline Casado/a & $20(44,4)$ & $25(55,6)$ & $45(45,5)$ & $1,14(0,55-2,37)$ & $1,36(0,60-3,04)$ \\
\hline \multicolumn{6}{|l|}{ Estudios } \\
\hline Primarios inacabado & $15(31,3)$ & $33(68,8)$ & $48(48,5)$ & $C R$ & $C R$ \\
\hline Enfermedad de Alzheimer & $19(38,0)$ & $31(62,0)$ & $50(50,5)$ & CR & CR \\
\hline Trastorno cognitivo no especificado & $9(50,0)$ & $9(50,0)$ & $18(18,2)$ & $1,43(0,55-3,69)$ & $1,63(0,55-4,83)$ \\
\hline Demencia vascular & $5(41,7)$ & $7(58,3)$ & $12(12,1)$ & $0,91(0,27-3,01)$ & $1,16(0,32-4,19)$ \\
\hline Demencia mixta/otra & $7(36,8)$ & $12(63,2)$ & $19(19,2)$ & $0,69(0,25-1,94)$ & $0,95(0,31-2,83)$ \\
\hline
\end{tabular}

CR: categoría de referencia; CV mb/b: calidad de vida muy buena/buena; $\mathrm{CV} \mathrm{n/m:} \mathrm{calidad} \mathrm{de} \mathrm{vida} \mathrm{normal/mala;} \mathrm{IC} \mathrm{95 \% :} \mathrm{intervalo} \mathrm{de} \mathrm{confianza} \mathrm{del} 95 \%$.

que su vida tiene sentido? Ambas con una escala con cinco opciones de respuesta: nada, un poco, normal, bastante o extremadamente.

- Una faceta del área de relaciones sociales: ¿en qué grado está satisfecho con sus relaciones personales? Muy insatisfecho, insatisfecho, normal, satisfecho o muy satisfecho.

Estudios cualitativos, usando grupos focales con personas con demencia leve-moderada, han mostrado que estos aspectos son importantes para la CV de dichas personas $[3,27,28]$.

Asimismo, se recogió información sociodemográfica: sexo, edad, estado civil y nivel de estudios.

\section{Análisis estadístico}

En el análisis estadístico principal se aplicó el modelo de la regresión logística ordinal (RLO) para estudiar la CV en una escala ordinal de cinco opciones. Después del análisis principal, se realizó un segundo análisis para estudiar la variable dependiente (CV global) dicotomizada; para ello, los participantes se dividieron en dos grupos: los que referían una
CV muy buena/buena y los que referían una CV nor$\mathrm{mal} / \mathrm{mala} / \mathrm{muy}$ mala. Posteriormente se realizó en ambos casos un análisis multivariado mediante el procedimiento por pasos, opción adelante $(p<0,05$ para entrada; $p<0,10$ para la eliminación del término). Los análisis estadísticos se realizaron con el programa STATA v. 11.0.

\section{Resultados}

\section{Características de los participantes}

Participaron 106 personas con demencia, pero se excluyó a 7 por no constar ningún tipo de diagnóstico. La muestra estuvo compuesta por 99 participantes: el 50,5\% tenía enfermedad de Alzheimer; el $12,1 \%$, demencia vascular; el 19,2\%, demencia mixta y otras, y el $18,2 \%$, trastorno cognitivo no especificado. La edad media fue de 78,9 años, en el 63,6\% de los casos se trataba de mujeres y el $45,5 \%$ de los participantes estaba casado. La mitad de los participantes refirió haber completado los estudios primarios (Tabla I). 


\section{Análisis principal}

En primer lugar se llevó a cabo el análisis mediante RLO univariante, utilizando como variable dependiente la $\mathrm{CV}$ global con cuatro niveles: muy buena $(7,1 \%)$, buena $(33,3 \%)$, normal $(41,4 \%)$ y mala $(18,2 \%)$, ya que ningún participante valoró su $\mathrm{CV}$ como muy mala. Tomando la enfermedad de Alzheimer como categoría de referencia, no se encontraron diferencias estadísticamente significativas en la autopercepción de CV entre los diferentes tipos diagnósticos. Tampoco se observaron diferencias significativas al estudiar las variables sociodemográficas. En la tabla I se recogen los resultados del análisis.

La tabla II muestra la información del análisis univariado con las variables que informan sobre la percepción del estado de salud, disfrute de la vida, sentido de la vida y satisfacción con las relaciones personales. El 48,5\% de los participantes puntuó su estado de salud de muy bueno/bueno; nadie puntuó su estado de salud de excelente. Las personas que tenían un estado de salud muy bueno/bueno tenían mejor CV que las personas que tenían un estado de salud regular/malo, con una odds ratio (OR) de 5,79 y un intervalo de confianza del 95\% (IC 95\%) de 2,56-13,09. La media de problemas crónicos de salud fue de 3,84 $\pm 2,3$. No se observó asociación entre el número de éstos y la CV. Los problemas de salud comunicados con mayor frecuencia ( $>40 \%$ ) fueron artrosis, hipertensión, depresión y problemas de visión. La media de síntomas depresivos fue significativamente inferior en personas que informaron de una CV muy buena/buena, en comparación con las que manifestaron una $\mathrm{CV}$ regular/mala $(p<0,01)$; un mayor número de síntomas depresivos se asoció con peor CV (OR = 0,75; IC 95\% = $0,66-0,85)$. De la misma manera, al considerar el GDS-15 como variable dicotómica (GDS- $15<6$ frente $\mathrm{a} \geq 6$ ), se observó que personas con depresión (GDS-15 $\geq 6$ ) tenían peor CV que personas sin depresión (GDS-15 < 6) (OR = 0,15; IC 95\% = 0,06$0,36)$. La puntuación media del IB fue de 77,4; el $79,8 \%$ de los pacientes eran independientes o tenían un grado de dependencia leve para realizar las actividades básicas de la vida diaria ( $\mathrm{IB} \geq 55$ ); no se observó asociación entre la $\mathrm{CV}$ y el estado funcional. En cuanto al grado de deterioro cognitivo, el 28,3\% presentaba una demencia leve (MMSE > 20). El análisis de RLO no mostró asociación entre el grado de deterioro cognitivo y la CV.

En relación con el resto de variables se evidenció, tal como muestran las OR y los IC $95 \%$, una asociación significativa de todas ellas con la CV. Así, las personas que disfrutaban de la vida bastante/extre- madamente tenían mejor $\mathrm{CV}$ que las que disfrutaban nada/poco/normal; las que encontraban mayor sentido a la vida tenían mejor CV que las que encontraban menor sentido y las que estaban más satisfechas con sus relaciones personales tenían mejor $\mathrm{CV}$ que las que estaban menos satisfechas.

En el análisis multivariado de RLO (Tabla III), las variables asociadas con la CV fueron un buen estado de salud, una mayor satisfacción con las relaciones personales y, de forma negativa, un mayor número de síntomas depresivos.

\section{Análisis de la variable dependiente calidad de vida dicotomizada}

La regresión logística binaria se realizó con la variable $\mathrm{CV}$ dicotomizada en $\mathrm{CV}$ muy buena/buena (40,6\%) y CV normal/mala (59,4\%). En las tablas I y II se muestran los resultados de los análisis. No se observó asociación entre la CV y los tipos diagnósticos de demencia, ni entre la CV y las variables sociodemográficas (Tabla I). Con relación al estado de salud y resto de variables de estudio, las personas que tenían un estado de salud muy bueno/bueno mostraban mejor CV que las personas con estado de salud regular/malo (OR = 5,55; IC 95\% = 2,2913,42); al aumentar el número de síntomas depresivos, la CV empeoraba (OR = 0,82; IC 95\% = 0,71$0,94)$; personas con depresión (GDS-15 $\geq 6$ ) tenían peor CV que las personas sin depresión (GDS-15 $<6$ ) $(\mathrm{OR}=0,26$; IC 95\% = 0,10-0,64); las personas que disfrutaban más de la vida $(\mathrm{OR}=4,12$; IC $95 \%=$ 1,71-9,95), las que encontraban mayor sentido a la vida $(\mathrm{OR}=3,96$; IC $95 \%=1,68-9,36)$ y las que estaban más satisfechas con sus relaciones personales $(\mathrm{OR}=3,97$; IC 95\% = 1,51-10,42) tenían mejor CV. No se encontraron asociaciones significativas entre CV y el resto de variables de estudio (Tabla II).

En el análisis multivariado de regresión logística binaria se introdujeron todas las variables y se duplicaron aquéllas de las que se disponía información cuantitativa categórica. Las variables asociadas con la CV fueron el estado de salud, la satisfacción con las relaciones personales, disfrutar de la vida y la gravedad de la demencia (MMSE $>20$ frente a MMSE < 21).

\section{Discusión}

El principal objetivo del presente estudio fue investigar la percepción de la CV en personas con demencia. Maximizar la CV es uno de los objetivos principales en esta población [4,12]. El 40,4\% pun- 
Tabla II. Información de salud, estado funcional, creencias y relaciones sociales; análisis univariado.

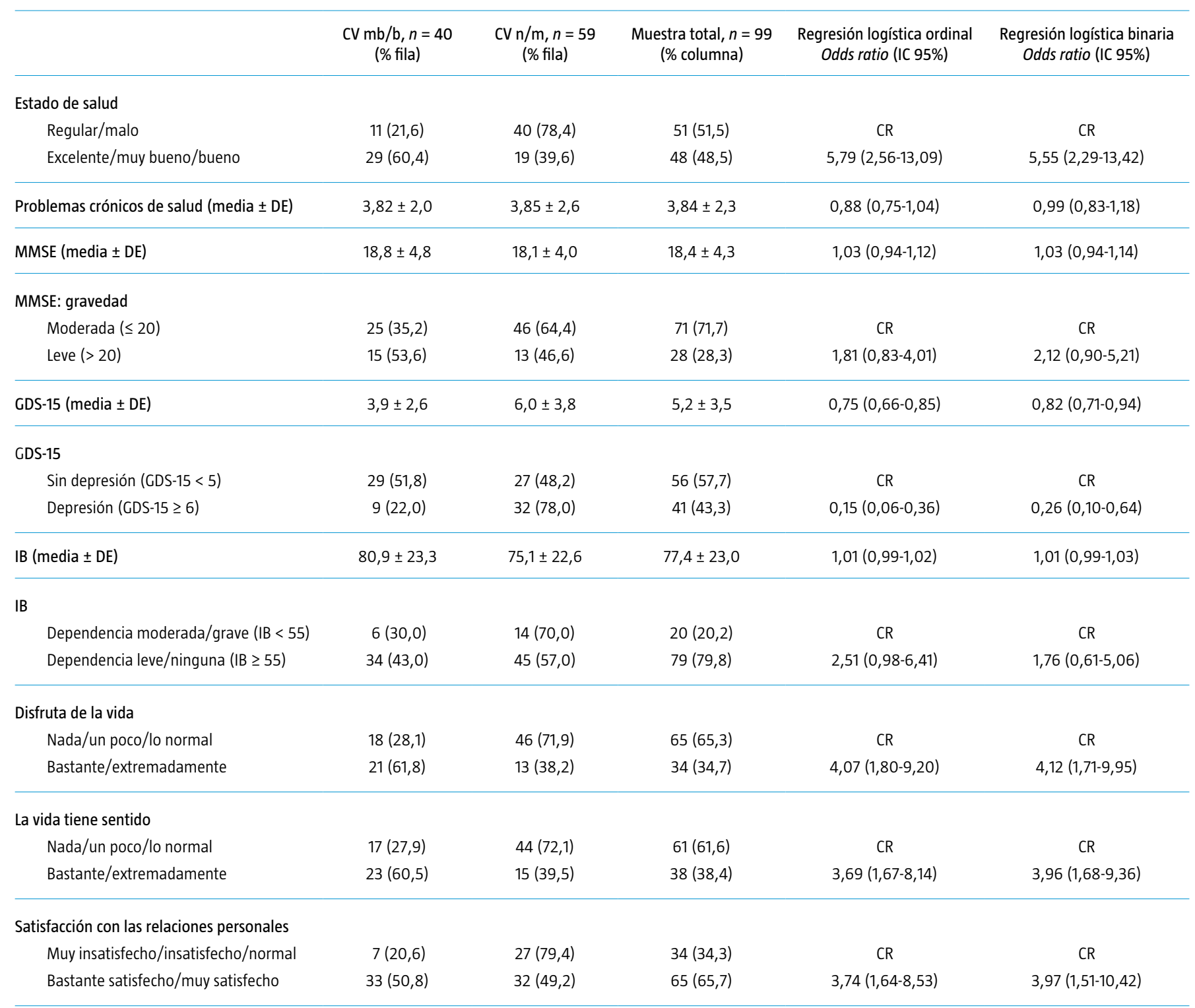

CR: categoría de referencia; CV mb/b: calidad de vida muy buena/buena; CV n/m: calidad de vida normal/mala; DE: desviación estándar; IC 95\%: intervalo de confianza del 95\%; CDS: escala de depresión geriátrica; IB: índice de Barthel; MMSE: Mini Mental State Examination.

tuó su CV global como muy buena/buena. No hemos encontrado estudios publicados en nuestro país relacionados con la autopercepción de $\mathrm{CV}$ en personas con demencia leve-moderada en los que se haya usado una pregunta global sobre CV. Este porcentaje es claramente inferior al $88 \%$ comunicado en el único estudio encontrado [17], que evalúa la $\mathrm{CV}$ global en personas con demencia con una única pregunta: ‘Cómo puntuaría su CV global en el momento actual? Excelente, muy buena, buena, regular o mala, y agrupada en excelente/muy buena/buena frente a regular/mala. Por tanto, una posible explicación es que la diferencia en el porcentaje de personas con demencia que informaron de una CV muy buena/buena sea debida a los descriptores de la escala de respuesta y agrupación poste- 
Tabla III. Factores significativos de los análisis multivariados.

\begin{tabular}{|c|c|c|}
\hline & Odds ratio (IC 95\%) & $p$ \\
\hline \multicolumn{3}{|l|}{ Regresión logística ordinal } \\
\hline Estado de salud ${ }^{a}$ & $4,80(1,98-11,62)$ & $<0,001$ \\
\hline Satisfacción con las relaciones personales ${ }^{\text {b }}$ & $6,47(2,53-16,53)$ & $<0,001$ \\
\hline GDS-15 & $0,78(0,68-0,89)$ & $<0,001$ \\
\hline \multicolumn{3}{|l|}{ Regresión logística binaria } \\
\hline Estado de salud a & $8,27(2,47-27,62)$ & 0,001 \\
\hline Satisfacción con las relaciones personales ${ }^{\text {b }}$ & $7,46(2,02-27,59)$ & 0,003 \\
\hline Disfruta de la vida c & $3,10(1,04-9,18)$ & 0,041 \\
\hline MMSEd $^{d}$ & $3,51(1,02-12,06)$ & 0,046 \\
\hline
\end{tabular}

a Excelente/muy buena/buena frente a regular/mala; ${ }^{b}$ bastante satisfecho/muy satisfecho frente a muy insatisfecho/insatisfecho/normal; ' ${ }^{c}$ bastante/extremadamente frente a nada/poco/normal; ${ }^{d}$ Mini Mental State Examination (MMSE): > 20 leve (21-30) frente a moderada (<21). GDS: escala de depresión geriátrica; IC 95\%: intervalo de confianza del $95 \%$. rior; sin embargo, el porcentaje encontrado sí es similar al observado en estudios realizados en personas mayores en nuestro medio, tanto en una muestra representativa de Cataluña [29] como en personas de centros de atención primaria [30], donde se utilizó la pregunta global del WHOQOL-BREF para evaluar la CV. La diferencia con el estudio de James et al [17] también pudo deberse a diferencias en otras variables; por ejemplo, utilizando el punto de corte convencional ( $\geq 6$ ) en la GDS-15 [25], el $43 \%$ de participantes presentaba depresión, porcentaje superior al 15,7\% comunicado por James et al [17]. La elevada prevalencia de síntomas depresivos o depresión en personas con demencia ha sido señalada en nuestro medio por otros autores [31-34]. La depresión se ha asociado de manera persistente con peor CV en personas mayores sin [33] y con trastornos cognitivos $[2,6,28]$.

Los porcentajes autocomunicados de problemas crónicos de salud (hipertensión, problemas de corazón, estómago, tiroides, visión y audición) fueron similares a los encontrados en personas mayores que acudían a centros de atención primaria [30]; sin embargo, las personas con demencia mostraron un mayor porcentaje de depresión, diabetes y problemas neurológicos. El mayor porcentaje de depresión autocomunicada (sí/no) concuerda con el elevado porcentaje encontrado de personas con depresión (GDS-15 $\geq 6$ ); las personas que manifestaron depresión presentaban mayor sintomatología depresiva que aquellas que indicaron que no tenían depresión $\left(\chi_{1 \mathrm{gl}}^{2}=10,32 ; p=0,001\right)$. Las personas que autocomunicaron problemas neurológicos principalmente refirieron enfermedad de Parkinson, demencia y/o problemas de memoria.

Ninguno de los análisis univariados mostró asociación entre la CV y el estado funcional (IB), la CV y la gravedad de la demencia (MMSE), ni entre la CV y las variables sociodemográficas. La falta de asociación entre CV y el estado funcional [2], y entre $\mathrm{CV}$ y variables sociodemográficas y gravedad de la demencia, se ha señalado previamente $[10,16,17$, $27,35,36]$. Un mejor estado de salud, un menor número de síntomas depresivos (GDS-15), una mayor satisfacción con las relaciones personales, encontrar sentido a la vida y disfrutar de la vida se asociaron con mejor CV. En ambos análisis multivariados, el estado de salud y la satisfacción con las relaciones personales permanecieron significativas. La salud es la variable que con mayor frecuencia se ha asociado con la CV o calidad de vida relacionada con la salud. Las relaciones personales se consideran importantes en algunas de las guías internacionales -por ejemplo, las del National Institute for Health and Clinical Excellence (NICE)- [37]; asimismo, su importancia se ha investigado mediante estudios cualitativos $[2,10,27,28,38]$. No obstante, se ha evidenciado escasamente con estudios cuantitativos. La asociación encontrada entre CV y sintomatología depresiva, en el análisis multivariado de RLO, se ha indicado previamente $[2,3,10,16]$.

En el análisis multivariado de regresión logística binaria, permanecen asociadas con la CV la gravedad de la demencia y disfrutar de la vida. Una menor gravedad de la demencia se asocia con mejor CV; sin embargo, esta asociación debe interpretarse con prudencia teniendo en cuenta su IC 95\% $(1,02-12,06)$. La mayoría de estudios señala una falta de asociación entre la gravedad de la demencia y la CV [6]. Solamente uno de los cuestionarios específicos de CV, el DEMQOL-28 [10] incluye 'disfrutar de la vida' como pregunta en una de sus áreas (sentimientos), pero no hemos hallado estudios cuantitativos que la analicen de manera independiente.

Disfrutar de la vida, encontrarle sentido a ésta y las relaciones personales son facetas pertenecientes a diferentes áreas (psicológica, creencias personales/espiritualidad y relaciones sociales) que forman parte del constructo multidimensional de $\mathrm{CV}$ tal como lo define la Organización Mundial de la Salud $[9,22,30]$. Estos aspectos son importantes y están asociados con la CV de personas adultas [21,22,39] y de personas mayores $[29,30,40]$. Nuestros resultados muestran que también siguen siendo impor- 
tantes y se asocian con la CV de personas con demencia leve-moderada. Así, se confirma nuestra hipótesis de que otros factores, y no sólo la gravedad de la demencia (MMSE), se asocian con la CV.

Respecto a limitaciones del estudio, los participantes no se seleccionaron de forma aleatoria; por tanto, los resultados no se pueden generalizar a toda la población de personas con demencia (incluyendo personas que no tienen un cuidador principal, personas que viven en residencias o con demencia en fases más avanzadas).

En resumen, nuestros resultados permiten afirmar que las personas con demencia leve-moderada son informantes adecuados de sus propios estados subjetivos, tanto de la percepción de su CV [17] como de su estado de salud [15], y que facetas hasta ahora omitidas en la evaluación de estas personas, como las relaciones personales y disfrutar de la vida, deben considerarse, tanto en la práctica clínica diaria como en ensayos clínicos, ya que son importantes y están asociadas con la CV $[27,28]$. Finalmente, para poder realizar comparaciones entre diferentes estudios, es necesario emplear escalas de respuesta que utilicen los mismos descriptores.

\section{Bibliografía}

1. World Health Organization, World Federation of Neurology. Atlas: country resources for neurological disorders. Geneva: WHO; 2004.

2. Banerjee S, Samsi K, Petrie CD, Alvir J, Treglia M, Schwam EM, et al. What do we know about quality of life in dementia? A review of the emerging evidence on the predictive and explanatory value of disease specific measures of health related quality of life in people with dementia. Int J Geriatr Psychiatry 2009; 24: 15-24.

3. Brod M, Stewart AL, Sands L, Walton P. Conceptualization and measurement of quality of life in dementia: the dementia quality of life instrument (DQoL). Gerontologist 1999; 39: 25-35

4. Rabins PV, Black BS. Measuring quality of life in dementia: purposes, goals, challenges and progress. Int Psychogeriatr 2007; 19: 401-7.

5. Winblad B, Brodaty H, Gauthier S, Morris IC, Orgogozo JM, Rockwood K, et al. Pharmacotherapy of Alzheimer's disease: is there a need to redefine treatment success? Int J Geriatr Psychiatry 2001; 16: 653-66.

6. Banerjee S, Smith SC, Lamping DL, Harwood RH, Foley B, Smith P, et al. Quality of life in dementia: more than just cognition. An analysis of associations with quality of life in dementia. J Neurol Neurosurg Psychiatry 2006; 77: 146-8.

7. Carr AJ, Higginson IJ. Are quality of life measures patient centred? BMJ 2001; 322: 1357-60.

8. Lawton MP. Quality of life in Alzheimer disease. Alzheimer Dis Assoc Disord 1994; 8: 138-50.

9. WHOQOL Group. The World Health Organization Quality of Life assessment (WHOQOL): position paper from the World Health Organization. Soc Sci Med 1995; 41: 1403-9.

10. Smith SC, Lamping DL, Banerjee S, Harwood R, Foley B, Smith P, et al. Measurement of health-related quality of life for people with dementia: development of a new instrument (DEMQOL) and an evaluation of current methodology. Health Technol Assess 2005; 9: 1-93.
11. Lucas-Carrasco R. Calidad de vida y demencia. Med Clin (Barc) 2007; 128: 70-5.

12. Karlawish JH, Casarett D, Klocinski J, Clark CM. The relationship between caregivers' global ratings of Alzheimer's disease patients' quality of life, disease severity, and the caregiving experience. J Am Geriatr Soc 2001; 49: 1066-70.

13. Vogel A, Mortensen EL, Hasselbalch SG, Andersen BB, Waldemar G. Patient versus informant reported quality of life in the earliest phases of Alzheimer's disease. Int J Geriatr Psychiatry 2006; 21: 1132-8.

14. Conde-Sala JL, Garre-Olmo J, Turró-Garriga O, López-Pousa $\mathrm{S}$, Vilalta-Franch J. Factors related to perceived quality of life in patients with Alzheimer's disease: the patient's perception compared with that of caregivers. Int J Geriatr Psychiatry 2009; 24: 585-94.

15. Waldorff FB, Nielsen AB, Waldemar G. Self-rated health in patients with mild Alzheimer's disease: baseline data from the Danish Alzheimer Intervention Study. Arch Gerontol Geriatr 2010; 50: 1-5.

16. Lucas-Carrasco R, Lamping DL, Banerjee S, Rejas J, Smith SC, Gómez-Benito J. Validation of the Spanish version of the DEMQOL system. Int Psychogeriatr 2010; 22: 589-97.

17. James BD, Xie SX, Karlawish JHT. How do patients with Alzheimer disease rate their overall quality of life? Am J Geriatr Psychiatry 2005; 13: 484-90.

18. American Psychiatric Association. Diagnostic and statistical manual of mental disorders. 4 ed. Washington DC: American Psychiatric Association; 1994.

19. Folstein MF, Folstein SE, McHugh PR. Mini mental state. A practical method for grading the cognitive state of patients for the clinician. J Psychiatr Res 1975; 12: 189-98.

20. Wilkinson D, Sganga A, Stave C, O'Connell B. Implications of the Facing Dementia Survey for health care professionals across Europe. Int J Clin Pract Suppl 2005; 146: 27-31.

21. WHOQOL Group. Development of the World Health Organization WHOQOL-BREF quality of life assessment. Psychol Med 1998, 28: 551-8.

22. Lucas-Carrasco R. La versión española del WHOQOL Madrid: Ergón; 1998

23. Mahoney FI, Barthel DW. Functional evaluation: the Barthel Index. Md State Med J 1965; 14: 61-5.

24. Baztán JJ, Pérez J, Alarcón T, San Cristóbal E, Izquierdo G, Manzarbeitia I. Îndice de Barthel: instrumento válido para la valoración funcional de pacientes con enfermedad cerebrovascular. Rev Esp Geriatr Gerontol 1993; 28: 32-40.

25. Yesavage JA, Brink TL, Rose TL, Lum O, Huang V, Adey M, et al. Development and validation of a geriatric depression screening scale: a preliminary report. J Psychiatr Res 1982; 17: 37-49.

26. Martí D, Miralles R, Llorach I, García-Palleiro P, Esperanza A, Guillem J, et al. Trastornos depresivos en una unidad de convalecencia: experiencia y validación de una versión española de 15 preguntas de la escala de depresión geriátrica de Yesavage. Rev Esp Geriatr Gerontol 2000; 35: 7-14.

27. Smith SC, Murray J, Banerjee S, Foley B, Cook JC, Lamping DL, et al. What constitutes health-related quality of life in dementia? Development of a conceptual framework for people with dementia and their carers. Int J Geriatr Psychiatry 2005; 20: 889-95.

28. Lucas-Carrasco R, Domencech-Pou S, Monteserín R. What constitutes quality of life for people with cognitive impairment and dementia? [abstract]. Qual Life Res Suppl 2007; A-12: abstract 1243.

29. Lucas-Carrasco R, March J, Salva-Casanovas A. Quality of life among elderly living in Catalonia, Spain [abstract]. Qual Life Res Suppl 2007; A-19: abstract 1621.

30. Lucas-Carrasco R. International perspective on quality of life in older adults. The WHOQOL OLD project. Vertex 2007; 18: $130-7$.

31. Contador-Castillo I, Fernández-Calvo B, Cacho-Gutiérrez LJ, Ramos-Campos F, Hernández-Martín L. Depresión en la demencia tipo Alzheimer: ¿existe algún efecto sobre la memoria? Rev Neurol 2009; 49: 505-10. 
32. Reyes-Figueroa JC, Rosich-Estragó M, Bordas-Buera E, Gaviria-Gómez AM, Vilella-Cuadrada E, Labad-Alqueza A. Síntomas psicológicos y conductuales como factores de progresión a demencia tipo Alzheimer en el deterioro cognitivo leve. Rev Neurol 2010; 50: 653-60.

33. Baquero M, Blasco R, Campos-García A, Garcés M, Fages EM, Andreu-Catalá M. Estudio descriptivo de los trastornos conductuales en el deterioro cognitivo leve. Rev Neurol 2004; 38: 323-6.

34. Dragomirecká E, Bartonová J, Eisemann M, Kalfoss M, Kilian R, Martiny K, et al. Demographic and psychosocial correlates of quality of life in the elderly from a cross-cultural perspective. Clin Psychol Psychother 2008; 15: 193-204.

35. Hurt CS, Banerjee S, Tunnard C, Whitehead DL, Tsolaki M, Mecocci P, et al. Insight, cognition and quality of life in Alzheimer's disease. J Neurol Neurosurg Psychiatry 2010; 81: 331-6.

36. Rosas-Carrasco O, Torres-Arreola L, Guerra-Silla MG,
Torres-Castro S, Gutiérrez-Robledo LM. Validación de la escala Quality of Life in Alzheimer's Disease (QOL-AD) en pacientes mexicanos con demencia tipo Alzheimer, vascular y mixta. Rev Neurol 2010; 51: 72-80.

37. National Institute for Health and Clinical Excellence. NICE clinical guideline 42. Dementia: supporting people with dementia and their careers in health and social care, 2006. URL: http://www.nice.org.uk/CG042fullguideline.

38. Ablitt A, Jones GV, Muers J. Living with dementia: a systematic review of the influence of relationship factors. Aging Ment Health 2009; 13: 497-511.

39. Power MJ, Bullinger M, Harper A, The WHOQOL Group. The World Health Organization WHOQOL-100: tests of the universality of quality of life in fifteen different cultural groups world-wide. Health Psychol 1999; 18: 495-505.

40. Power M, Quinn K, Schmidt S, The WHOQOL-Olf Group. Development of the WHOQOL-Old module. Qual Life Res 2005; 14: 2197-214.

\section{Overall quality of life in persons with dementia}

Aim. To assess overall quality of life (QoL) among persons with dementia and to investigate variables associated with subjective perception of QoL.

Patients and methods. This was a cross-sectional study in six health and social care centres in Barcelona and Tarragona. Ninety nine persons with mild-moderate dementia, living at home with a known caregiver completed a face-to-face interview including sociodemographic information; health perception, a list of chronic medical conditions, depressive symptoms and activities of daily living. Overall QoL was measured with the single item of the World Health Organization Quality of Life-BREF.

Results. 40.6\% assessed their overall QoL as very good/good. Univariate logistic regression analyses showed a lack of association between QoL with sociodemographic information, severity of dementia and functioning, but significant association between QoL with depressive symptoms, satisfaction with relationships, enjoying life and meaning in life. Ordinal and binary multivariate regression analysis showed that better perception of health and greater satisfaction with personal relationships was associated with better QoL. In addition, fewer depressive symptoms in the ordinal multivariate regression analysis and dementia severity and enjoy life in the binary regression analysis appeared significant.

Conclusions. Persons with mild-moderate dementia can inform about their overall QoL and subjective states. In addition to health status, other dimensions, as personal relationships and positive aspects should be systematically assessed in this population.

Key words. Dementia. Health status. Quality of life. Self assessment. 